\title{
OVERCOMING AUGMENTED REALITY ADOPTION BARRIERS IN DESIGN: A MIXED PROTOTYPING CONTENT AUTHORING TOOL SUPPORTED BY COMPUTER VISION
}

\author{
Piñones, Eduardo; \\ Cascini, Gaetano; \\ Caruso, Giandomenico; \\ Morosi, Federico \\ Politecnico di Milano
}

\begin{abstract}
Enhancing the appearance of physical prototypes with digital elements, also known as mixed prototyping, has demonstrated to be a valuable approach in the product development process. However, the adoption is limited also due to the high time and competence required for authoring the digital contents. This paper presents a content authoring tool that aims to improve the user acceptance by reducing the specific competence required, which is needed for segmentation and UV mapping of the 3D model used to implement a mixed prototype. Part of the tasks related to 3D modelling software, in fact, has been transferred to simpler manual tasks applied onto the physical prototype. Moreover, the proposed tool can recognise these manual inputs thanks to a computer-vision algorithm and automatically manage the segmentation and UV mapping tasks, freeing time for the user in a task that otherwise would require complete engagement. To preliminarily evaluate effectiveness and potential of the tool, it has been used in a case study to build up the mixed prototype of a coffee machine. The result demonstrated that the tool can correctly segment the 3D model of a physical prototype in its relevant parts and generate their corresponding UV maps.
\end{abstract}

Keywords: New product development, Computational design methods, Industrial design, Mixed prototyping, Augmented reality

\section{Contact:}

Piñones Zuleta, Eduardo Andrés

Politecnico di Milano

Mechanical engineering

Italy

eduardo.pinones@polimi.it

Cite this article: Piñones, E., Cascini, G., Caruso, G., Morosi, F. (2021) 'Overcoming Augmented Reality Adoption Barriers in Design: A Mixed Prototyping Content Authoring Tool Supported by Computer Vision', in Proceedings of the International Conference on Engineering Design (ICED21), Gothenburg, Sweden, 16-20 August 2021. DOI:10.1017/ pds. 2021.497 


\section{INTRODUCTION}

Prototypes are fundamental in the product development process as different types of prototypes can take different roles depending on the needs of the developers and the expected decision outcomes (Zhou and Rau, 2019). In this context, prototypes supported by projector-based Augmented Reality (AR), also known as mixed prototypes due to their ability to simultaneously include features from a physical and virtual prototype, have allowed developers to create fully functional prototypes without the need of electronics, displays or physical buttons (Marner et al., 2011), create on-the-fly perceptual deformation and surface material manipulation during design evaluation (Takezawa et al., 2019), and enhance communication during co-creative design sessions (O'Hare et al., 2018) among other benefits, which in conjunction speed-up the design iterations and reduce the cost of prototyping in the final stages of development.

Nevertheless, mixed prototyping and AR technologies in general are still not widely adopted within product development processes as several reasons impact negatively the user acceptance, including lack of realism in the surface reproduction (Cascini et al., 2020), high requirements of competence for content authoring (Bhattacharya and Winer, 2019) and a challenging set-up process for running the mixed prototyping platform consistently (Giunta et al., 2019). Although research is being carried out to solve some of those problems, there is a discrepancy between the research and industrial interests, being most of the research focused on technological challenges, overlooking organizational challenges, while they are equally relevant for the industry. This difference of interests becomes even more noticeable in regard of user acceptance (Masood and Egger, 2019). Due to this situation, currently content authoring depends mostly on the developers of the technology or IT experts, and therefore the scale-up becomes difficult due to time requirements and content creation costs (Masood and Egger, 2020), counteracting some of the main benefits of mixed prototyping.

To properly take advantage of this technology and improve user acceptance, attention should be put on easing the content authoring process. As stated by previous research, there is the need to develop innovative tools that are more intuitive to create the augmented experience (de Souza Cardoso et al., 2020) and research should focus on the implementation, keeping in mind the intelligence requirements of the manufacturing industry of the future (Egger and Masood, 2020). Correspondingly, the motivation of this paper concerns the implementation of a new approach to ease content authoring for mixed prototyping, focusing on one of the stages of the process that at the same time: (i) is time consuming; (ii) requires high-level competencies; and (iii) is independent of the mixed prototyping platform used. More specifically, the focus of this work is on the segmentation of the 3D model into parts that should be manipulated separately in the AR application and on their UV mapping, a process required to transfer $2 \mathrm{D}$ information (e.g. textures or other graphical elements) to a $3 \mathrm{D}$ model and ultimately project them on a physical prototype.

The contribution of the paper is to present an innovative content authoring tool for mixed prototyping content authoring aiming at reducing the competencies requirements and improving the user acceptance of this technology. The paper begins with an analysis of previous research on easing content authoring for mixed prototyping and AR technologies, as well as the relevance of 3D modelling within the authoring process and suitable approaches. A generic workflow for content authoring, the proposed approach and an illustrative case within a product development process are then presented. The paper concludes with a discussion on the results and limitations of the proposed approach as well as future work and testing to be carried out.

\section{BACKGROUND}

\subsection{Content authoring for mixed prototyping}

Since mixed prototyping is in early stages of development (Cascini et al., 2020), no standard method supports the complete workflow of content authoring on all implementations. Nevertheless, as presented in Figure 1, a general workflow that generates a linkage between a physical prototype and a 3D model to create a usable mixed prototype can still be outlined. 


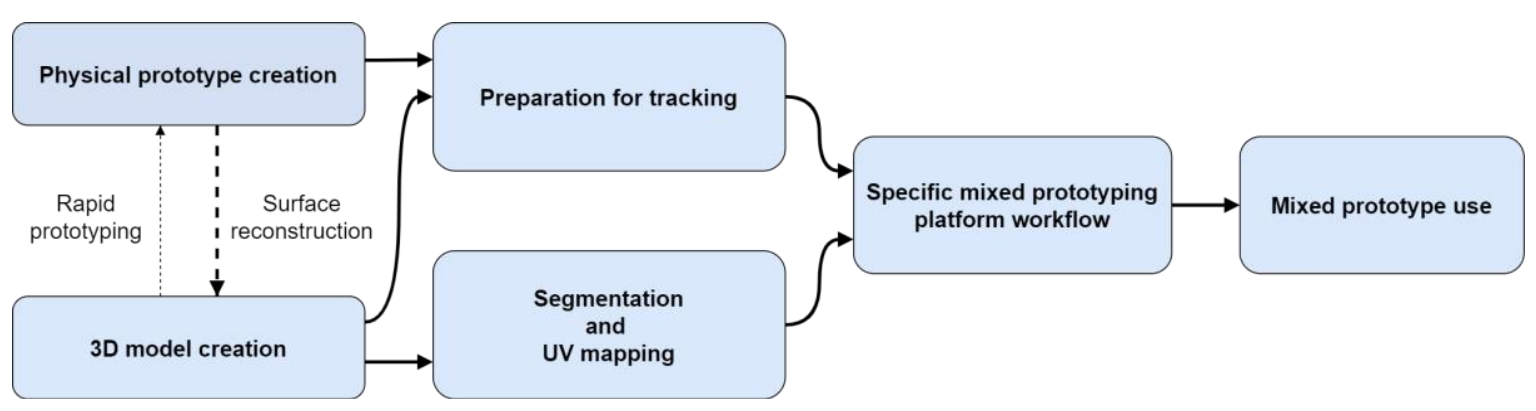

Figure 1. General workflow of content authoring for mixed prototyping

In mixed prototyping, a 3D model of the physical prototype or of the relevant parts to be tested, is necessary to correctly project the virtual information over its surface and depending on which of them is created first the workflow can vary slightly. On one side, if the 3D model is created first, the physical prototype can be manufactured e.g. through rapid prototyping technologies, and on the other side, if the physical prototype is created first then the 3D model can be created e.g. through computeraided design. While in some cases the production and alignment of the physical and digital model is not a problem, not all development processes require 3D modelling competence, as packaging industry, fashion and accessories, orthopaedic braces just to mention a few. Furthermore, some prototypes manufactured through manual techniques will not correspond to the correspondent digital models due to geometrical imperfections and some prototypes that are not completely solid will change their shape after user interaction. Considering those limitations, a larger diffusion of AR applications in product development requires a method and supporting tools capable of creating digital models of prototypes at a low cost, with minimal competence by the user and in a restricted timeframe. In this perspective, suitable opportunities derive from surface reconstruction methods such as photogrammetry (Misal et al., 2019), a process in which several pictures of an object from different perspectives are analysed to estimate its shape and generate a textured 3D model.

Regardless of the method used to create the 3D model of the physical prototype, AR applications also need to segment the digital model into several parts that are going to receive a different treatment, e.g. to augment the corresponding portions of the physical model with diverse textures, annotations and interaction modalities. Moreover, mapping bidimensional graphical elements on the surface of the physical object requires correlating the coordinates of the $2 \mathrm{D}$ object and the $3 \mathrm{D}$ coordinates of the vertices and polygons that make up the 3D model (UV mapping).

In addition, to enable the user to physically interact with the mixed prototype (e.g. moving it to see different perspectives) and coherently map the digital content in real-time, a tracking technology, which streams data of the position and rotation of the object, is needed. Tracking technologies include the use of computer vision to detect fiducial markers, infrared markers and even using natural features detection of the object as input information to obtain a pose estimation (van Lopik et al., 2020). After the segmentation of the 3D model, the UV mapping and the preparation for tracking, the model is ready for the integration in the mixed prototyping platform. Some other sub-steps such as calibration of the system, definition of graphical assets and creation of an initial design layout could be required according to the technologies and functionalities of the platform (O'Hare et al., 2018).

\subsection{Content authoring methods on similar applications}

In previous research on AR applied in design tasks, the content has been mostly created by the developers and not by the final users (Masood and Egger, 2020). This limitation has been also identified by several AR and virtual reality implementations in fields like architecture, engineering and construction, where the upskilling of the users is one of the main barriers for their successful adoption (Davila et al., 2020). Efforts have been done to ease the content authoring process of AR applications for assembly and maintenance, two of the most researched fields on industrial applications of AR (Egger and Masood, 2020). Such solutions include using depth-sensing cameras to overcome occlusion problems and speed-up the creation process (Gimeno et al., 2013), retargeting existing printed technical documentation to AR (Mohr et al., 2015) and the use of template-based formatting combined with eye-tracking to record processes and create AR content (van Lopik et al., 2020). However, no solution was found to be directly applicable to mixed prototyping as they focus on aiding the execution of a process and not the capability to edit in real time the digital content of the mixed 
prototype, which is a key feature to adopt AR in design activities. Nevertheless, solutions focused on at least one of the initial steps of general workflow of content authoring for mixed prototyping (as in Figure 1) could still ease the process as a whole and improve user acceptance as they do not depend directly on the mixed prototyping platform to be used.

From the initial steps, the segmentation and UV mapping stand out with the highest priority for improvement, as they are time consuming and requiring specific competence in 3D modelling (Tarini et al., 2017), and in principle the development of supporting tools might be exploited also in other virtual reality applications, 3D rendering etc. In other fields where segmentation and UV mapping have been a required skill for years, such as game development and visual effects, several automation methods have been proposed, including the minimization of angle deformation on the 3D mesh of separated parts (Lévy et al., 2002), the use of concavities in the mesh as an input to separate them into meaningful components (Katz and Tal, 2003) and the optimization of texture distortion on the created UV map (Poranne et al., 2017). Nevertheless, most of them focus on the use of geometric information to segment the 3D model, an approach that is not applicable at least in two opposite circumstances:

- a prototype where one independent part has a complex geometry and still should not be segmented into different patches;

- a prototype whose geometry is as simple as a brick or a cylinder and still one might want to segment it into several parts to be treated in a different manner.

On a different approach, in the mixed prototyping workflow, if 3D models created by surface reconstruction methods are already textured, this information could be used to guide the segmentation and UV mapping process, an option that is overlooked by the previously mentioned techniques. Moreover, extra information could be manually embedded in the prototype surface before scanning to further improve the usability of the texture, being later analysed through computer vision techniques to extract relevant features (e.g., crevices on the physical prototype, markers or added lines) and correlate them to vertices in the $3 \mathrm{D}$ model with minimal user intervention. Although this is a promising approach, its implementation is still challenging as several difficulties must be overcome to properly detect those features and correlate them to the $3 \mathrm{D}$ model, such as variations in the texture colours due to illumination during scanning, imperfections on manually added features and exclusion of not relevant features. Moreover, since surface reconstructed models usually have a high number of polygons, up to millions if needed, several polygons can be correlated to a detected feature, and therefore, if that feature delimits the boundaries between different parts, then the selection of the optimal boundary path should be addressed as well.

\section{THE PROPOSED TOOL AND ITS WORKFLOW}

This section presents an integrated workflow of content authoring for mixed prototyping spanning from the availability of a physical prototype to the exporting of a 3D model suitable for mixed prototyping that is properly segmented and UV mapped. To aid this process, a tool was developed under the assumption that the user knows at least how to import a 3D model in a 3D modelling software and navigate in the viewport. More specifically, an algorithm was developed for Blender, an open-source 3D modelling software, being programmed using an internal implementation of Python as well as functions of OpenCV, an external library for computer vision.

As mentioned in the previous section, the segmentation and UV mapping of the 3D model is a required step for virtually any system with $3 \mathrm{D}$ rendering capabilities. However, since each platform for mixed prototyping still can have its own workflows, some steps of the procedure here described, not strictly related to segmentation and UV mapping, could vary. To have a realistic view of the implementation, some steps of the workflow have been adapted to the SPARK project, a mixed prototyping solution supported by projector-based augmented reality focused on co-creative design sessions (Cascini et al., 2020).

\subsection{Physical prototype preparation}

Considering that a physical prototype is already available, the surface should be prepared to reproduce the projected virtual information as intended in terms of colour accuracy. To do so, a homogeneous paint coat of a light neutral colour such as white or grey should be applied. It is worth mentioning that some solutions to calibrate the colour reproduction independently of the surface colour have been proposed, nevertheless a proper preparation of the surface reduce the need of these corrective measures. 
After the surface has been prepared, extra information to aid the segmentation and UV mapping can be added to the surface manually. More specifically, black lines are manually drawn over the physical prototype marking the boundaries of each independent part. Special attention should be put to ensure the generation of closed boundaries to avoid that two contiguous parts are detected as one. Moreover, depending on the mixed prototyping platform, other information could be added at this stage. In the case of SPARK, retro-reflective infrared markers needed for tracking must be applied to get a reference of their position in the 3D model and ease calibration.

\subsection{Surface reconstruction}

Once the physical prototype has been prepared, a 3D model must be generated. Several techniques and off-the-shelf 3D scanners are suitable for this process. However, the main requirement is that the selected method can generate a corresponding texture for the 3D model as this information will be needed to correlate the added surface features to the vertices and polygons in the 3D model. Photogrammetry is a suitable approach for this task as several commercial software are available, allowing to create 3D models of prototypes at a low cost and with minimal competence. Although, it is worth noting that while surface reconstruction methods can produce a 3D model that have the correct proportions of the scanned object, the scale or a rotation are not necessarily correct. Similarly, surface reconstruction software creates an initial UV map to apply the textures on the 3D model. However, this UV map is not suitable for a mixed prototype due to the reasons mentioned in section 2.2.

\subsection{Preparation of 3D model for projection system}

Using the results from the previous step, the textured 3D model is imported into Blender, where the algorithm adjusts the rotation, scale and location of the 3D model to match the physical prototype dimensions and ease the projection calibration.

To adjust the rotation, the algorithm moves the 3D model in the middle of the screen with an orthographic front view. A panel is also displayed with the options necessary to change the rotation of the 3D model around the three principal axes, as well as the option to change to different viewpoints. This allows aligning the base of the object with the corresponding plane, obtaining a 3D model with the same orientation as the physical prototype over a surface.

To adjust the scale, the algorithm requests to place two markers on the surface of the 3D model and input the distance value between those two points in the physical prototype. The distances in the 3D model and the physical prototype are then compared and the dimension of the 3D model is modified to match the physical prototype.

Moreover, for the specific tracking solution of the SPARK project, the origin (i.e., a point in the 3D space that defines the location of the 3D model) should be equal to one of the previously added retroreflective infrared markers. Since the location of the markers is embedded in the texture, the algorithm requests to click on the desired marker and automatically relocates the origin to it.

\subsection{Extraction of texture features}

As shown in Figure 2, to extract the added features from the original texture the algorithm executes a series of processing steps. The algorithm accesses the existing data of the 3D model and stores the original texture (a) while at the same time uses an internal function of Blender to generate an image of the initial UV map (b). The adaptive thresholding function from the OpenCV library is then used to extract the lines from the original texture (c). This function generates a new image with the same dimensions as the original texture in which a colour black or white is assigned to each pixel depending on the contrast with the pixels around it, in this way slight variations on the colours of the original texture due to illumination changes during scanning will not affect the detection of the lines. As a result, an image is generated with high contrast features (e.g., added lines) marked white and the rest of the surface marked black. However, other details in the texture that have high contrast with the surface (e.g., dirt) and small sections of the texture generated by the automated UV mapping of the surface reconstruction software will be assigned as white pixels as well. A second image is generated using the threshold function on the initial UV map (d), from which any pixel group that is too small to correspond to a line is removed (e). The result of this step is then used as a mask (f) to discard any part of the texture that is outside of the UV map boundaries as well as remove unwanted elements. 
Moreover, since the dimensions of the original texture, the width of the lines in the physical prototype and imperfections in the surface reconstruction process could affect the proper detection of the lines, two parameters can be controlled. First, the amount of neighbour pixels that are used during the adaptive thresholding, where a higher value allows the detection of wider lines in terms of the number of pixels. Second, the possibility to apply a dilation function to the extracted lines (g), a function that expands the selection of pixels marked as white, solving possible discontinuities where a section of the line was not properly detected but also widening the line selection, which increases the processing times during the segmentation of the 3D model.

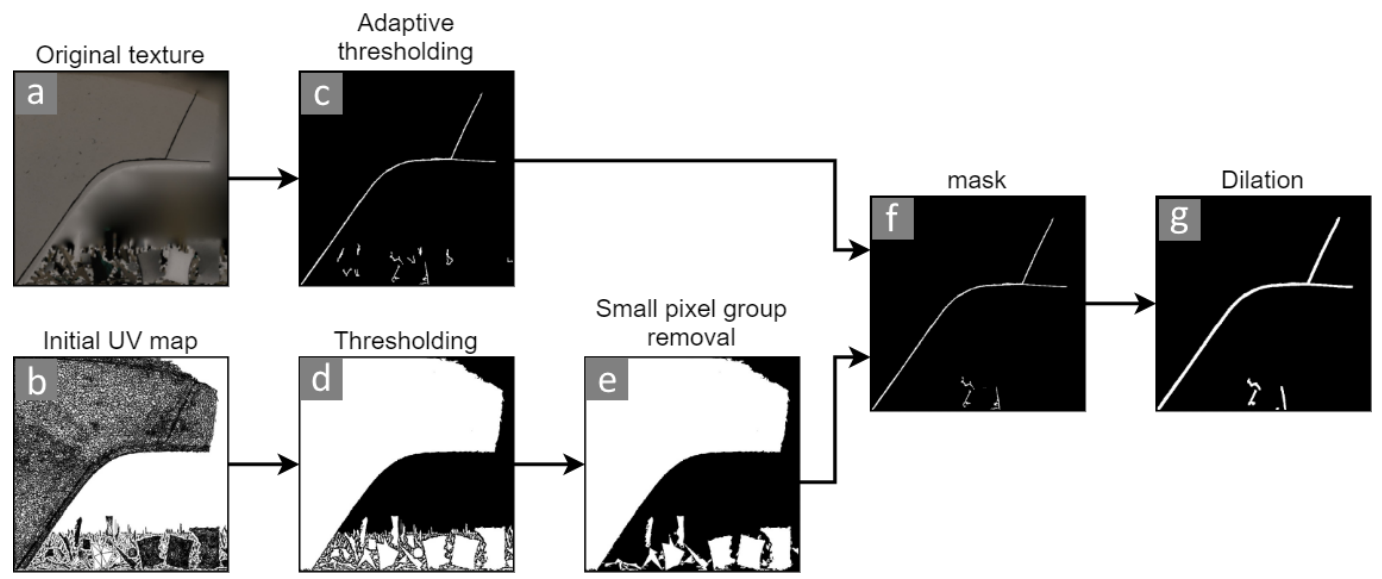

Figure 2. Processing steps to extract added features from the original texture

Once the lines have been properly extracted from the original texture, an internal function of Blender is used to access the coordinates of each vertex in the UV map generated by the surface reconstruction software, each of those coordinates is then correlated to the coordinates of a pixel in the image of the extracted lines. Using this information, a list of all the vertices that were positioned in a white pixel within the image of extracted lines is stored.

\subsection{Segmentation of 3D model and UV mapping}

Using the information obtained in the previous step, the algorithm segments all the polygons of the 3D model that are completely composed by vertices within the stored list. This process generates several 3D meshes which, as shown in Figure 3, will be separated into two categories for processing purposes. If the $3 \mathrm{D}$ mesh is composed by multiple polygons, it is assumed that the mesh corresponds to the surface of a part of the prototype and will be called primary mesh, being the number of the primary meshes equal to the number of segmented parts in the result. If the 3D mesh is composed by an individual polygon, is assumed that it was positioned inside a line and will be called secondary mesh. Nevertheless, to avoid that small primary meshes generated by imperfections in the selection are considered as independent parts of the prototype, the algorithm allows controlling the maximum number of the primary meshes either by selecting beforehand the number of final parts and keeping the meshes with the highest number of polygons or by setting the number of polygons below which a separated mesh will be considered a secondary mesh.

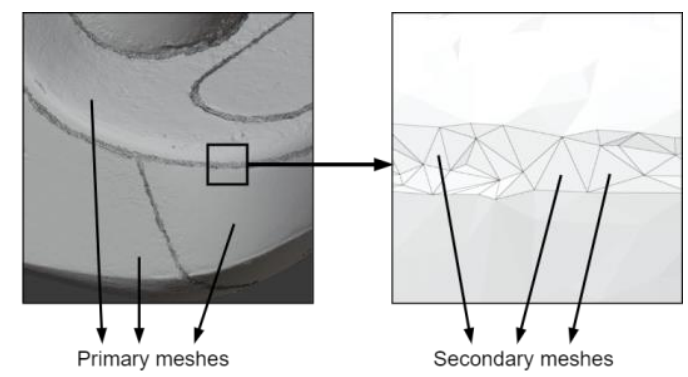

Figure 3. Categorization of segmented meshes

In general terms, each secondary mesh should be assigned and joined to the nearest primary mesh resulting in a 3D model properly separated. However, due to the high number of polygons in 3D 
models generated through surface reconstruction, it is likely that in the width of each line many secondary meshes could be found. In turns, it is not a trivial task to decide to which primary mesh assign each secondary mesh. To address this issue, for each secondary mesh the position of its vertices inside a white pixel within the image of extracted lines is checked while an erosion function is iteratively applied to the image (See figure 4). This function, contrary to the dilation function, reduces the size of the areas with pixels marked as white, which, in practical terms, allows progressively reducing the width of the detected lines from both sides, and therefore once one of the vertices of the secondary mesh is positioned outside the line (i.e., in a black pixel), it is possible to assign and join the secondary mesh to the primary mesh whose boundaries are near to the vertex that was outside the line.

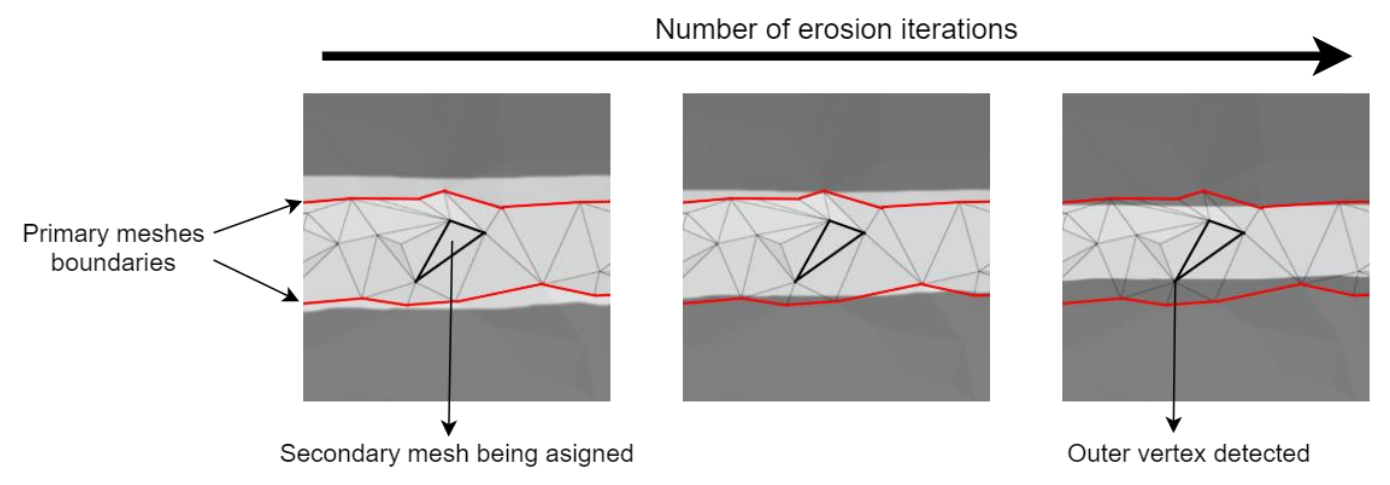

Figure 4. separated model (a), generated UV maps (b) and results on MR platform (c)

Once all the secondary meshes have been assigned, the 3D model will be separated in multiples parts with reduced geometric complexity in comparison to the initial 3D model, and therefore automated UV mapping solutions can now be used for each primary mesh and the complete model can be exported to be used in a mixed prototyping platform, a process implemented using internal functions of Blender.

\section{ILLUSTRATIVE CASE AND DISCUSSION}

To illustrate the proposed content authoring tool, the physical prototype of a coffee machine was used as a base to create a mixed prototype suitable for the mixed prototyping platform supported by projector-based augmented reality and focused on co-creative design sessions. Although this was a simulated case, the selected prototype was useful from two perspectives. First, to test a product on which two common tasks of the product development process can be assessed: material selection and design of user interfaces. And second, to test the robustness of the tool due to the complex geometry and multiple parts of the prototype.

Figure 5 shows the physical prototype already prepared with the added superficial features (a) (i.e., lines and retro-reflective infrared markers) as well as the results of the surface reconstruction (b), where can be seen that the added features have been properly transferred to the texture of the 3D model (c). The 3D model was composed of 149,959 vertices and 299,914 polygons.

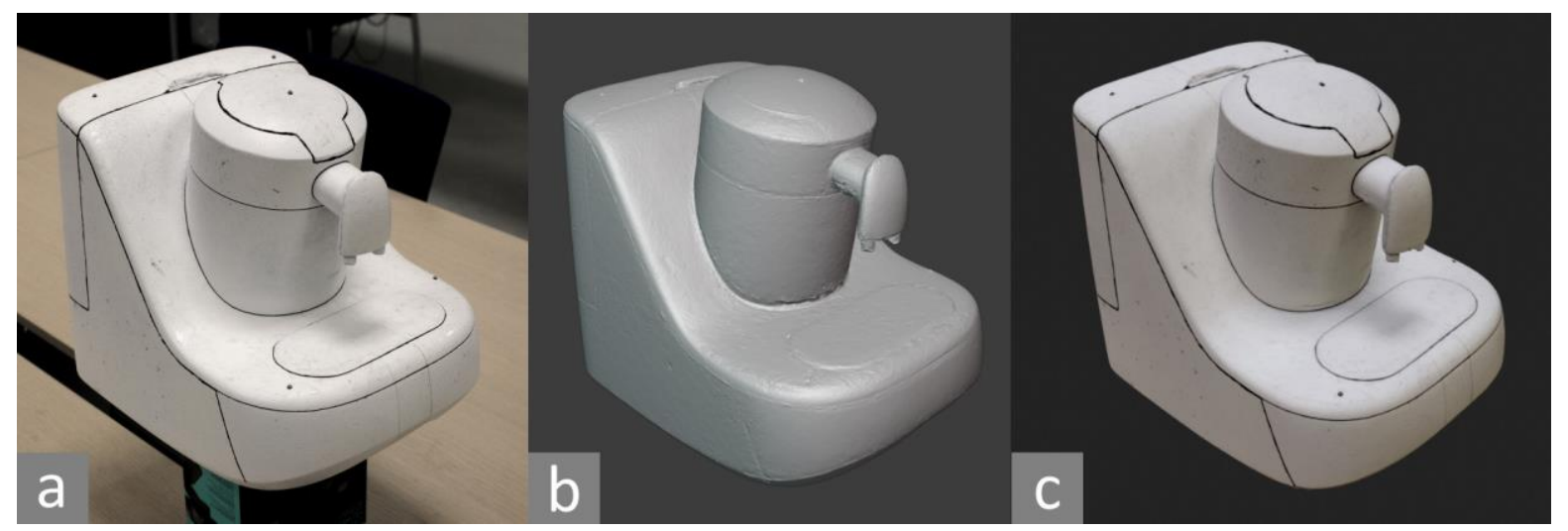

Figure 5. Picture of the physical prototype (a), reconstructed mesh generated through photogrammetry (b) and textured $3 D$ model imported in Blender (c) 
Once the textured 3D model was imported in Blender, its rotation and scale of the model were adjusted and the origin of the 3D model was relocated using the developed algorithm. Figure 6 shows the results of the first three steps of the preparation of the 3D model for real-time editing: the extraction of the original texture (a) and initial UV map (b) generated by the surface reconstruction software, the resulting extracted lines from the original texture (c) and the selection of vertices in the 3D model based on the extracted lines (d).

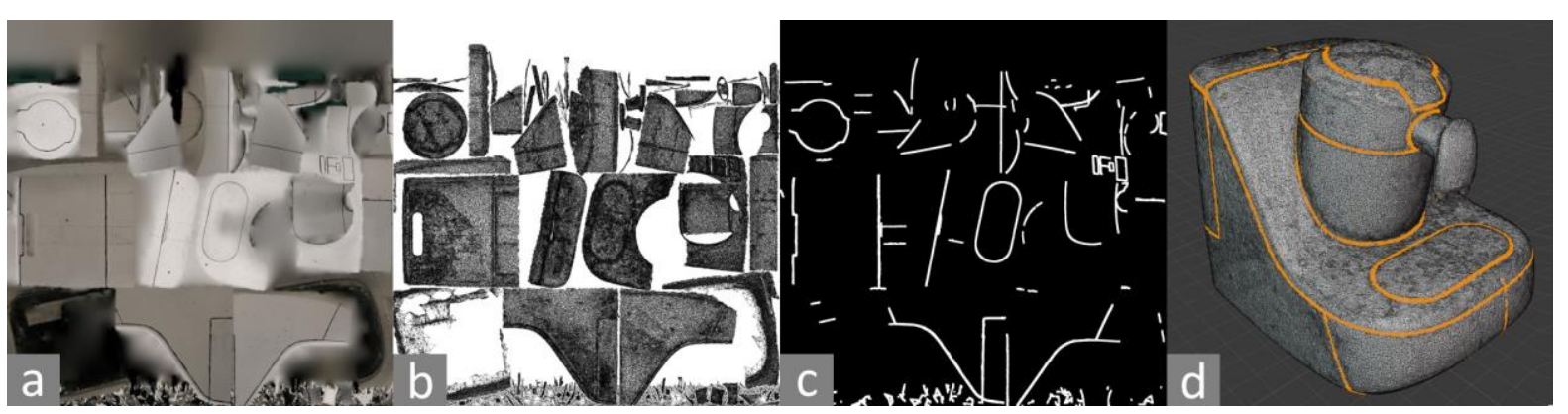

Figure 6. Original texture (a), initial UV map (b), extracted lines (c) and selected vertices (d)

Finally, Figure 7 shows the results of the segmentation of the 3D model (a) and UV mapping of the individual parts (b), as well as the use of those results in the mixed prototyping platform, where two different design variants characterized by different look and interaction elements were simulated (c \& d). The process of segmentation was executed with a user input of number of final parts expected equal to 12 , with which a total of 33,142 polygons were categorized as secondary meshes, and the total processing time of this step was 24 minutes and 46 seconds using a computer with an Intel Core i7$9750 \mathrm{H}$ processor.
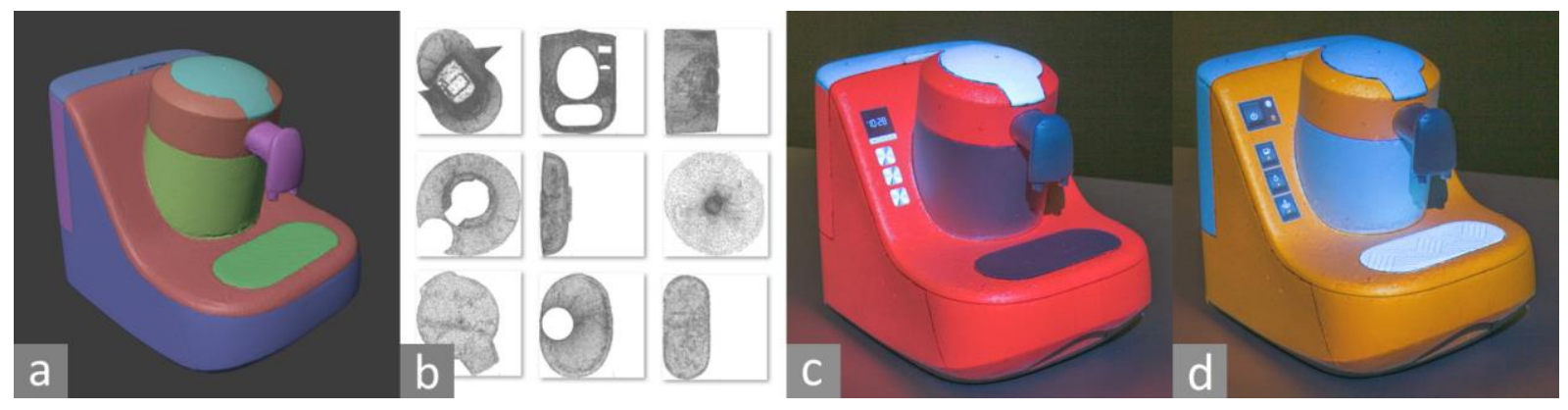

Figure 7. separated model (a), generated UV maps (b) and design variants projected over a physical prototype $(c \& d)$

\subsection{Discussion}

From the technical perspective, as shown in the illustrative case, the proposed tool supported by computer vision is a suitable approach for mixed prototyping content authoring, as it can separate the 3D model on the relevant parts of the prototype and generate the corresponding UV maps for real-time interactions. Nevertheless, some limitations arise during testing, including the relatively long time of processing required for segmentation of the 3D model and UV mapping, which does not allow on-thefly preparation of a mixed prototype within a design session and thus could negatively affect the user acceptance of this workflow. However, the proposed approach turns out to be much more convenient with respect to the current process, which takes a similar amount of time but require specific competence and the user must remain engaged in the activity during the whole process to obtain the same results (an estimation by the author is that an experienced user would manually dedicate 20 to 40 minutes in the segmentation and UV mapping of the illustrative case).

Moreover, due to the irregular 3D mesh generated by the surface reconstruction software, the boundaries of the separated parts were not defined following a straight path, affecting the quality of the projection. Although this effect was almost no noticeable in the illustrative case due to the high number of polygons of the exported 3D model, it could become more relevant for mixed prototyping platforms supported by mobile applications, where the number of polygons must be reduced due to performance restriction. 
From the user experience perspective, the decision of how to separate the 3D model is transferred from a task in a 3D modelling software to a task in the preparation of the physical prototype. Therefore, the competence requirements on 3D modelling during the execution of the process are reduced in comparison with the process done manually; a change that is expected to positively influence the user acceptance and the technology adoption of mixed prototyping technologies enabled by AR (de Souza Cardoso et al., 2020).

In the product development process, the proposed content authoring workflow could improve the adoption of mixed prototyping and its benefits in initial design stages, where currently other prototyping methods that require lower 3D modelling competences are preferred. Moreover, in cases where mixed prototyping is already a suitable option, the proposed workflow has the potential to increase the flexibility to apply geometrical changes in the prototype during design sessions, an option that is limited by the necessity of specialized users to create a new content (Bhattacharya and Winer, 2019).

\subsection{Future work}

To evaluate the impact and limitations of this research to improve the user acceptance of mixed prototyping technologies, further testing stages are required. Being relevant to obtain a realistic comparison with the current status of content authoring, the testing activity will involve the target users of this tool such as employees of design agencies and users with competence in 3D modelling capable of separating the 3D model of the prototype and create the corresponding UV maps by means of the default functionalities implemented in a 3D modelling software. Some of the parameters of comparison in which the testing should focus are:

- User acceptance.

- $\quad$ Time required to prepare a 3D model suitable for mixed prototyping starting from an existing physical prototype.

- Complexity of the process in terms of number of tasks and the level of competence required to execute each of them.

- Workload during the preparation of the mixed prototype.

- Precision of the boundaries in the separated parts of the 3D model.

- Quality of the created UV maps in terms of the mount of distortion in the final projected image.

Moreover, although the results obtained during the illustrative case seem sufficient to be used in its current state, further improvements in the developed tool should be also considered, as it can directly affect some of the parameters mentioned before. More specifically, improving performance of the algorithm for segmentation and UV mapping can reduce the total time required to prepare mixed prototyping content. The tool should also consider some options to improve the boundary selection on $3 \mathrm{D}$ models with a lower number of polygons or alternatively optimize the $3 \mathrm{D}$ model before it is exported, as this will expand the possible uses also to platforms supported by mobile devices.

\section{CONCLUSIONS}

With the final aim of increasing the adoption of mixed prototyping, the work reported in this paper illustrates and discusses the viability of a tool for mixed prototyping content authoring supported by computer vision focused on reducing competence requirements and improve user acceptance. The proposed tool was able to separate the $3 \mathrm{D}$ model on the relevant parts of the prototype and generate the corresponding UV maps for real-time interactions, one of the stages of the process that is at the same time: time consuming, have high competence requirements and is required independently of the mixed prototyping platform used. Moreover, the decision of how to separate the 3D model was transferred from a task in a $3 \mathrm{D}$ modelling software to a task in the preparation of the physical prototype, lowering the competence requirements in 3D modelling, and as a result potentially increasing the number of users that have currently the required competences to create content for mixed prototyping. While competence requirement is not the only factor that could affect mixed prototyping adoption within the product development process, it is a step forward to lower the current barriers.

Nevertheless, this initial research does not address the further impacts and limitations of the proposed workflow in comparison with a content authoring process executed using the current tools that $3 \mathrm{D}$ modelling software provide, being necessary further testing also including the participation of target users. 


\section{REFERENCES}

Bhattacharya, B. and Winer, E.H. (2019), "Augmented reality via expert demonstration authoring (AREDA)", Computers in Industry, Vol. 105, pp. 61-79. https://doi.org/10.1016/j.compind.2018.04.021

Cascini, G., O’Hare, J., Dekoninck, E., Becattini, N., Boujut, J.-F., Ben Guefrache, F., Carli, I., et al. (2020), "Exploring the use of AR technology for co-creative product and packaging design", Computers in Industry, Vol. 123, p. 103308. https://doi.org/10.1016/j.compind.2020.103308

Davila, J.M., Oyedele, L., Demian, P. and Beach, T. (2020), “A research agenda for augmented and virtual reality in architecture, engineering and construction”, Advanced Engineering Informatics, Vol. 45, p. 101122. https://doi.org/10.1016/j.aei.2020.101122

Egger, J. and Masood, T. (2020), “Augmented reality in support of intelligent manufacturing - A systematic literature review”, Computers \& Industrial Engineering, Vol. 140, pp. 106-195. https://doi.org/10.1016/j.cie.2019.106195

Gimeno, J., Morillo, P., Orduña, J.M. and Fernández, M. (2013), “A new AR authoring tool using depth maps for industrial procedures”, Computers in Industry, Vol. 64 No. 9, pp. 1263-1271. https://doi.org/10.1016/j.compind.2013.06.012

Giunta, L., Guefrache, F.B., Dekoninck, E., Gopsill, J., O’Hare, J. and Morosi, F. (2019), “Investigating the Impact of Spatial Augmented Reality on Communication between Design Session Participants - A Pilot Study", Proceedings of the Design Society: International Conference on Engineering Design, Cambridge University Press, Vol. 1 No. 1, pp. 1973-1982. https://doi.org/10.1017/dsi.2019.203

Katz, S. and Tal, A. (2003), "Hierarchical mesh decomposition using fuzzy clustering and cuts", ACM Transactions on Graphics (TOG), ACM. https://dl.acm.org/doi/abs/10.1145/882262.882369

Lévy, B., Petitjean, S., Ray, N. and Maillot, J. (2002), "Least squares conformal maps for automatic texture atlas generation", ACM Transactions on Graphics, Vol. 21 No. 3, pp. 362-371. https://doi.org/10.1145/566654.566590

van Lopik, K., Sinclair, M., Sharpe, R., Conway, P. and West, A. (2020), "Developing augmented reality capabilities for industry 4.0 small enterprises: Lessons learnt from a content authoring case study", Computers in Industry, Vol. 117, p. 103-208. https://doi.org/10.1016/j.compind.2020.103208

Marner, M.R., Smith, R.T., Porter, S.R., Broecker, M.M., Close, B. and Thomas, B.H. (2011), "Large Scale Spatial Augmented Reality for Design and Prototyping", in Furht, B. (Ed.), Handbook of Augmented Reality, Springer New York, New York, NY, pp. 231-254. https://doi.org/10.1007/978-1-4614-0064-6_10

Masood, T. and Egger, J. (2019), “Augmented reality in support of Industry 4.0_-Implementation challenges and success factors”, Robotics and Computer-Integrated Manufacturing, Vol. 58, pp. 181-195. https://doi.org/10.1016/j.rcim.2019.02.003

Masood, T. and Egger, J. (2020), “Adopting augmented reality in the age of industrial digitalisation”, Computers in Industry, Vol. 115, pp. 103-112. https://doi.org/10.1016/j.compind.2019.07.002

Misal, Khan, I.M., Babar, M. and Mudassir, M.U. (2019), "Implementation of 3D Scanning Technique for Object Model Replication and Customization Using Rapid Prototyping", 2019 International Conference on Engineering and Emerging Technologies (ICEET), pp. 1-6. https://doi.org/10.1109/CEET1.2019.8711836

Mohr, P., Kerbl, B., Donoser, M., Schmalstieg, D. and Kalkofen, D. (2015), "Retargeting Technical Documentation to Augmented Reality", Proceedings of the 33rd Annual ACM Conference on Human Factors in Computing Systems, Association for Computing Machinery, New York, NY, USA, pp. 33373346. https://doi.org/10.1145/2702123.2702490

O’Hare, J., Dekoninck, E., Giunta, L., Masclet, C. and Cascini, G. (2018), "Exploring the performance of augmented reality technologies in co-creative sessions: Initial results from controlled experiments", presented at the 5th International Conference on Design Creativity. https://doi.org/10.21278/idc.2018.0391

Poranne, R., Tarini, M., Huber, S., Panozzo, D. and Sorkine-Hornung, O. (2017), “Autocuts: simultaneous distortion and cut optimization for UV mapping”, ACM Transactions on Graphics, Vol. 36 No. 6. https://doi.org/10.1145/3130800.3130845

de Souza Cardoso, L.F., Mariano, F.C.M.Q. and Zorzal, E.R. (2020), “A survey of industrial augmented reality”, Computers \& Industrial Engineering, Vol. 139, p. 106-159. https://doi.org/10.1016/j.cie.2019.106159

Takezawa, T., Iwai, D., Sato, K., Hara, T., Takeda, Y. and Murase, K. (2019), "Material Surface Reproduction and Perceptual Deformation with Projection Mapping for Car Interior Design”, 2019 IEEE Conference on Virtual Reality and 3D User Interfaces (VR), pp. 251-258. https://doi.org/10.1109/VR.2019.8797923.

Tarini, M., Yuksei, C. and Lefebvre, S. (2017), "Rethinking Texture Mapping", presented at the SIGGRAPH, Los Angeles. https://doi.org/10.1145/3084873.3084911

Zhou, X. and Rau, P.-L.P. (2019), "Determining fidelity of mixed prototypes: Effect of media and physical interaction”, Applied Ergonomics, Vol. 80, pp. 111-118. https://doi.org/10.1016/j.apergo.2019.05.007 